\title{
A physical mechanism based investigation on the elasto-plastic-damage behavior of anisotropic aluminum alloys under finite deformation
}

\author{
CHEN Cen ${ }^{1 *}$, LIANG NaiGang ${ }^{1}$, LIU Fang $^{2} \&$ FU Qiang ${ }^{3}$ \\ ${ }^{1}$ State Key Laboratory of Nonlinear Mechanics, Institute of Mechanics, Chinese Academy of Sciences, Beijing 100190, China; \\ ${ }^{2}$ School of Transportation, Wuhan University of Technology, Wuhan 430063, China; \\ ${ }^{3}$ China National Light Weight Gas Turbine Development Center, Beijing 100190, China
}

Received June 3, 2013; accepted October 23, 2013; published online January 22, 2014

\begin{abstract}
The elasto-plastic-damage behavior of anisotropic aluminum alloys is investigated under finite deformation using a physical mechanism based constitutive model. With an application to the structural calculation, the present model is used to describe and analyze the mechanical response of anisotropic 6260-T6 aluminum alloy extrusions. For the tensile specimens extracted along three different material orientations from the extruded aluminum profile, twelve simulations are carried out covering four different specimen geometries. The simulation results in force-displacement response and central logarithmic axial strain evolution are compared with experimental results. From the comparisons, it can be concluded that the present model has the capacity to describe the behavior of anisotropic material. From the force-displacement curves, the anisotropy is observed in different material orientations, and the physical mechanism of anisotropy is analyzed.
\end{abstract}

\section{elasto-plastic-damage behavior, anisotropy, physical mechanism, finite deformation, constitutive theory, component assembling model}

PACS number(s): 46.35.+z, 46.70.De, 46.05.+b

Citation: Chen C, Liang N G, Liu F, et al. A physical mechanism based investigation on the elasto-plastic-damage behavior of anisotropic aluminum alloys under finite deformation. Sci China-Phys Mech Astron, 2014, 57: 400-410, doi: 10.1007/s11433-013-5381-1

\section{Introduction}

In the sheet forming process, the sheets usually possess different properties in rolling and transverse extrusion orientations. The effect of anisotropy causes difficulties in determining the mechanical behavior of materials, especially under the condition of plastic deformation and damage induced anisotropy. In order to solve this problem, many efforts have been put into the study of anisotropic behavior of materials.

When it comes to plastic deformation, classical plasticity

*Corresponding author (email: chencen@lnm.imech.ac.cn) theory and crystal plasticity theory investigate the plastic anisotropy in different ways. As a macroscopic approach, classical plasticity theory is inseparable from the yield function. Hill [1,2] proposed an anisotropic yield function with six parameters in the definition of an equivalent stress. As an important extension, the Yld2000-2d yield function was proposed by Barlat et al. [3-6]. Recently, Dunand et al. [7] extended the Yld2000-2d model to three dimensional stress states and investigated the anisotropic behavior of aluminum alloy extrusions. In addition, great progress has been made in the microscopic perspective of crystal plasticity theory. For example, the Taylor-type crystal plasticity developed by Garmestani et al. [8] and the crystal plasticity 
model with anisotropic strain hardening rule proposed by Zamiri et al. [9].

For the damage stage, continuum damage mechanics $(\mathrm{CDM})$ and microscopic damage mechanics are intended to study the anisotropic damage from different viewpoints, and damage variable is an important base to describe the damage for many models. In the CDM, based on the scalar damage variables for isotropic damage model [10], vectors [11] or tensors [12-15] were incorporated into damage models to describe the anisotropic damage. On the other hand, microscopic damage mechanics is employed to obtain the macroscopic response from the microstructural physical process involved in microcrack growth, and several important micromechanical damage models were proposed [16-19].

There are two approaches mentioned above: macroscopic and microscopic. Both of them have their advantages and disadvantages: macroscopic approach allows for a direct implementation in the Finite Element Method (FEM) codes, but it can't connect the texture evolution to the anisotropy of the materials. On the other hand, microscopic approach can explain the material behavior based on the texture evolution, but it is difficult to implement in the FEM program.

So combination of the two approaches is a promising path to construct applicable elasto-plastic damage models with a solid physical foundation. In recent years, progress has been made in developing material models by incorporating microscopic information. For example, Peeters et al. [20] and Kowalczyk et al. [21] attempted to describe the evolution of macroscopic plastic anisotropy based on the microstructure of polycrystals, and implemented their models in numerical simulations. On the other hand, some models based on interatomic potentials were proposed to reflect the microstructure of materials. The Virtual Internal Bond (VIB) model [22] and the Quasicontinuum model [23] described the mechanical response of atoms within the framework of continuum mechanics. The physical mechanism based model is not only easy to apply in structural calculation, but also able to explain the material behavior.

However, some basic requirements should be considered for establishing a constitutive model [24,25]. For example, it must be objective. So an appropriate expression for constitutive equation under finite deformation is very important [26]. And more requirements should be considered for the complicated behaviors in the deformation processes of some materials [27]. So, we also hope the constitutive model has the following characteristics: firstly, it can describe the mechanical behavior of whole deformation process including elasticity, plasticity and damage; then, it has the capacity to describe the anisotropy and complicated deformation under finite deformation.

In this paper, the mechanical behavior of anisotropic aluminum alloy is investigated using a physical mechanism based elasto-plastic damage constitutive model named component assembling model. The model is assembled by the spring-bundle, cubage and slip components. The springbundle and cubage components are abstracted from pair functional potentials to describe the elasto-damage constitutive relation. Considering that slip is the main plastic deformation mechanism of polycrystalline metals, we propose slip component to describe the plastic behavior of material. Based on the decomposition of total deformation into elastic deformation and plastic deformation, the elasto-plastic damage constitutive relation has been established by assembling the responses of three kinds of components.

Based on the present model, the constitutive equation is implemented into the finite element simulation tool ABAQUS. For the extruded 6260-T6 aluminum alloy, simulations are performed based on the experiments of Luo et al. [28] and Dunand et al. [7]. With the comparisons between simulation and experimental results, the physical mechanism of anisotropy is analyzed.

\section{Material model}

The component assembling model was proposed by Deng et al. [29-31] in the infinitesimal deformation. Zhang et al. [32] studied the damage induced anisotropy of quasi-brittle materials based on this model. Liu et al. [33,34], Fu et al. [35,36] and Chen et al. [37] extended it into the condition of finite deformation and investigated the evolution of subsequent yield surface. In this paper, only essential parts of derivation process for the material model under finite deformation are provided.

\subsection{Elasto-damage constitutive relation}

Based on pair functional potentials and Cauchy-Born rule [38], the atomic bonds in the same direction are represented by a spring-bundle component, and the atom embedding interaction is represented by a cubage component. The spring-bundle components are discrete uniformly all over the space and reflect the pair potential. The only one cubage component reflects the embedding potential. The elastic deformation and the damage of material can be described by spring-bundle and cubage components, and the elastodamage constitutive equation is established by the Jaumann rate of Kirchhoff stress $\stackrel{\nabla}{\tau\left(w^{e}\right)}$ and the symmetric rate of elastic stretching tensor $\boldsymbol{D}^{e}[34]$ :

$$
\stackrel{\nabla}{\boldsymbol{\tau}\left(\boldsymbol{W}^{e}\right)}=\boldsymbol{C}^{e}: \boldsymbol{D}^{e}
$$

where, $\boldsymbol{W}^{e}$ is the elastic part of lattice spin tensor, $\boldsymbol{C}^{e}$ is the tangent elasto-damage stiffness tensor. Its components are expressed as: 


$$
\begin{aligned}
C_{i j k l}^{e}= & \frac{1}{2} \sum_{s=1}^{n} f\left(\lambda^{(s)}\right) \mathrm{e}^{-2 \lambda^{(s)}}\left(n_{j}^{(s)} n_{l}^{(s)} \delta_{i k}+n_{i}^{(s)} n_{k}^{(s)} \delta_{j l}\right. \\
& \left.+n_{j}^{(s)} n_{k}^{(s)} \delta_{i l}+n_{i}^{(s)} n_{l}^{(s)} \delta_{j k}\right) \\
& +\sum_{s=1}^{n}\left(f^{\prime}\left(\lambda^{(s)}\right)-2 f\left(\lambda^{(s)}\right)\right) \mathrm{e}^{-4 \lambda^{(s)}} n_{i}^{(s)} n_{j}^{(s)} n_{k}^{(s)} n_{l}^{(s)} \\
& +p^{\prime}(\theta) \delta_{i j} \delta_{k l},
\end{aligned}
$$

where, $\lambda^{(s)}$ is the deformation of a spring-bundle component, $f\left(\lambda^{(\mathrm{s})}\right)$ is the force response of the spring-bundle component, $\boldsymbol{n}^{(s)}$ is the direction vector, and $n_{i}^{(s)}$ is the component of $\boldsymbol{n}^{(s)} ; \theta$ is the deformation of cubage component, $p(\theta)$ is the force response of the cubage component. $f^{\prime}\left(\lambda^{(s)}\right)$ and $p^{\prime}(\theta)$ are the tangent stiffness of spring-bundle component and cubage component respectively. $\delta_{i j}$ is the Kronecker delta.

Since the essence of damage is the decrease and loss of atomic bonding forces, the damage effect can be reflected by the force response of spring-bundle and cubage components. For simplicity, the damage of cubage component is not considered in the present work.

\subsection{Elasto-plastic damage constitutive relation}

Dislocation slip is considered as the main plastic deformation mechanism of most metals. By extending crystal plasticity to polycrystalline, we propose the slip component to describe plastic deformation. The response of slip component under finite deformation is similar to that of crystal plasticity.

The slip components are uniformly discrete in the space. For the slip component $\alpha$, the kinematic variable is shearing deformation $\gamma^{(\alpha)}$ and the kinetic variable is resolved shear stress $\tau^{(\alpha)}$. According to the generalized Schmid law, for a potentially active or critical slip component, the resolved shear stress must reach its critical value. For a continuously active slip component, the resolved shear stress must increase to and remain at the critical value. It is assumed that the rate of critical resolved shear stress is linearly related to the shearing deformation rate on slip components:

$$
\left\{\begin{array}{l}
\dot{\tau}^{(\alpha)}=\dot{\tau}_{+c r}^{(\alpha)}=\sum_{\beta=1}^{m} h_{\alpha \beta} \dot{\gamma}^{(\beta)}, \dot{\tau}_{-c r}^{(\alpha)}=b \dot{\tau}_{+c r}^{(\alpha)}, \sum_{\beta=1}^{m} h_{\alpha \beta} \dot{\gamma}^{(\beta)}>0, \\
\dot{\tau}^{(\alpha)}=\dot{\tau}_{-c r}^{(\alpha)}=\sum_{\beta=1}^{m} h_{\alpha \beta} \dot{\gamma}^{(\beta)}, \dot{\tau}_{+c r}^{(\alpha)}=b \dot{\tau}_{-c r}^{(\alpha)}, \sum_{\beta=1}^{m} h_{\alpha \beta} \dot{\gamma}^{(\beta)}<0,
\end{array}\right.
$$

where, $h_{\alpha \beta}$ is the hardening modulus matrix and reflects the affect of component $\alpha$ on component $\beta$. $b$ is the Bauschinger effect parameter that determines the influence of positive critical resolved shear stress on the negative one. When $\sum_{\beta} h_{\alpha \beta} \dot{\gamma}^{(\beta)}>0$, the positive critical resolved shear stress $\tau_{+c r}^{(\alpha)}$ changes due to hardening, the negative one $\tau_{-c r}^{(\alpha)}$ changes by Bauschinger effect, and vice versa.
The Jaumann rate of Kirchhoff stress is written as:

$$
\begin{aligned}
{ }_{\boldsymbol{\tau}}^{\nabla} & =\boldsymbol{C}^{e}:\left(\boldsymbol{D}-\boldsymbol{D}^{p}\right)-\sum_{\alpha=1}^{m} \dot{\gamma}^{(\alpha)}\left(\boldsymbol{Q}^{(\alpha)} \cdot \boldsymbol{\tau}-\boldsymbol{\tau} \cdot \boldsymbol{Q}^{(\alpha)}\right) \\
& =\left(\boldsymbol{C}^{e}-\sum_{\alpha=1}^{m} \sum_{\beta=1}^{m}\left(g_{\alpha \beta}\right)^{-1} \boldsymbol{\lambda}^{(\alpha)} \otimes \boldsymbol{\lambda}^{(\beta)}\right): \boldsymbol{D},
\end{aligned}
$$

where, $\boldsymbol{D}$ is the symmetric rate of stretching tensor, and $\boldsymbol{D}^{p}$ is the symmetric rate of plastic stretching tensor. $\tau$ is Kirchhoff stress and the sum runs over the active slip components. $\quad \boldsymbol{\lambda}^{(\alpha)}=\boldsymbol{P}^{(\alpha)}: \boldsymbol{C}^{e}+\boldsymbol{Q}^{(\alpha)} \cdot \boldsymbol{\tau}-\boldsymbol{\tau} \cdot \boldsymbol{Q}^{(\alpha)}, \quad$ and $\quad g_{\alpha \beta}=$ $h_{\alpha \beta}+\lambda^{(\alpha)}: \boldsymbol{P}^{(\beta)}$. $\boldsymbol{P}^{(\alpha)}$ and $\boldsymbol{Q}^{(\alpha)}$ are the symmetric and antisymmetric tensor respectively: $\boldsymbol{P}^{(\alpha)}=\frac{1}{2}\left(\boldsymbol{s}^{(\alpha)} \otimes \boldsymbol{n}^{(\alpha)}+\boldsymbol{n}^{(\alpha)}\right.$ $\left.\otimes \boldsymbol{s}^{(\alpha)}\right), \quad \boldsymbol{Q}^{(\alpha)}=\frac{1}{2}\left(\boldsymbol{s}^{(\alpha)} \otimes \boldsymbol{n}^{(\alpha)}-\boldsymbol{n}^{(\alpha)} \otimes \boldsymbol{s}^{(\alpha)}\right)$. For the slip component $\alpha, s^{(\alpha)}$ is the vector in the slip direction and $n^{(\alpha)}$ is the vector in the normal direction of slip plane.

Defining $\boldsymbol{C}^{\text {epd }}$ as the elasto-plastic damage stiffness tensor by

$$
\boldsymbol{C}^{e p d}=\boldsymbol{C}^{e}-\sum_{\alpha=1}^{m} \sum_{\beta=1}^{m}\left(g_{\alpha \beta}\right)^{-1} \boldsymbol{\lambda}^{(\alpha)} \otimes \boldsymbol{\lambda}^{(\beta)},
$$

where, the components of elasto-damage stiffness tensor $\boldsymbol{C}^{e}$ are determined by eq. (2).

More details about the material model were given by Liu et al. [34].

\section{Finite element formulation}

For the structural calculation based on the FEM, the constitutive equation (4) would be transformed to a linear format using the update Lagrange method. In the calculation, the last deformed state is defined as the reference configuration.

According to the update Lagrange method, the second Piola-Kirchhoff stress $\boldsymbol{T}$ is equal to the Kirchhoff stress $\tau$ in the reference configuration. In the current configuration, the relationship between the material spin of Kirchhoff stress $\dot{\tau}$ and that of the second Piola-Kirchhoff stress $\dot{\boldsymbol{T}}$ can be expressed as:

$$
\dot{T}_{i j}=\dot{\tau}_{i j}-L_{i p} \tau_{p j}-\tau_{i p} L_{j p}
$$

where, $L$ is the velocity gradient tensor.

The components of the Jaumann rate of Kirchhoff stress can be written as:

$$
{ }^{\nabla} \tau_{i j}=\dot{\tau}_{i j}+\tau_{i p} \cdot W_{p j}-W_{i p} \cdot \tau_{p j},
$$

where, $W$ is the lattice spin, and it is an antisymmetrical tensor. So the material spin of Kirchhoff stress $\dot{\tau}$ can be written as: 


$$
\begin{aligned}
\dot{\tau}_{i j}= & \tau_{i j}+\tau_{i p} \cdot W_{j p}+W_{i p} \cdot \tau_{p j} \\
= & \tau_{i j}+\tau_{i p} \cdot\left(L_{j p}-D_{j p}\right)+\left(L_{i p}-D_{i p}\right) \cdot \tau_{p j} \\
= & \left(C_{i j k l}^{e p d}-\tau_{i k} \delta_{j l}-\tau_{k j} \delta_{i l}\right) D_{k l}+L_{j p} \tau_{i p}+L_{i p} \tau_{p j},
\end{aligned}
$$

where, the components of elasto-damage stiffness tensor $C_{i j k l}^{e p d}$ are calculated by eq. (5).

Substituting eq. (8) into eq. (6) yields,

$$
\dot{T}_{i j}=\left(C_{i j k l}^{e p d}-\tau_{i k} \delta_{j l}-\tau_{k j} \delta_{i l}\right) D_{k l}
$$

The relationship between the rate of Green strain $\dot{\boldsymbol{E}}$ and the symmetric rate of stretching tensor $D$ is expressed as:

$$
\dot{\boldsymbol{E}}=\boldsymbol{F}^{\mathrm{T}} \cdot \boldsymbol{D} \cdot \boldsymbol{F}
$$

where, $\boldsymbol{F}$ is deformation gradient.

Since the last deformed state is defined as the reference configuration, the principal elongation ratio is regarded as 1 in the current configuration. Then the rate of Green strain $\dot{\boldsymbol{E}}$ is equal to the symmetric rate of stretching tensor $D$, i.e. $\dot{\boldsymbol{E}}=\boldsymbol{D}$ [25], eq. (9) can be expressed as:

$$
\Delta T_{i j}=\left(C_{i j k l}^{e p d}-\tau_{i k} \delta_{j l}-\tau_{k j} \delta_{i l}\right) \cdot \Delta E_{k l} .
$$

The constitutive equation is transformed into a linear format as:

$$
\Delta \boldsymbol{T}=\tilde{\boldsymbol{C}}: \Delta \boldsymbol{E}
$$

where $\tilde{\boldsymbol{C}}$ is the stiffness tensor that satisfies the character of Voigt symmetry, and its components can be written as:

$$
\tilde{C}_{i j k l}=C_{i j k l}^{e p d}-\tau_{i k} \delta_{j l}-\tau_{k j} \delta_{i l}
$$

\section{Iteration procedure}

The linear constitutive equation (11) is integrated into FEM simulation tool ABAQUS. For each integration point, the increase of strain is given and the stress is calculated in the user subroutine VUMAT. The whole calculation procedure in an increment step is presented as:

(1) The increase of Green strain $\Delta \boldsymbol{E}^{(n)}$ is given.

(2) Since $\boldsymbol{D}^{(n)}=\Delta \boldsymbol{E}^{(n)}$ holds in the update Lagrange method, the symmetric rate of elastic stretching tensor is calculated by $\left(\boldsymbol{D}^{e}\right)^{n}=(\boldsymbol{D})^{n}-\left(\boldsymbol{D}^{p}\right)^{n-1}$. The initial value of $\boldsymbol{D}^{p}$ is set as 0 .

(3) The increase of elastic deformation gradient is computed by $\Delta \boldsymbol{F}^{e}=\left(\boldsymbol{D}^{e}+\boldsymbol{W}^{e}\right) \cdot\left(\boldsymbol{F}^{e}\right)^{n-1}$; the value of $\boldsymbol{W}^{e}$ is set as 0 . The total elastic deformation gradient is $\left(\boldsymbol{F}^{e}\right)^{n}=\Delta \boldsymbol{F}^{e}+\left(\boldsymbol{F}^{e}\right)^{n-1}$.

(4) The deformations of spring-bundle and cubage components can be obtained by the elastic deformation gradient $\left(\boldsymbol{F}^{e}\right)^{n}[34]$, and the force responses of these components would be calculated.

(5) The elasto-damage stiffness tensor $\boldsymbol{C}^{e}$ is obtained by eq. (2).

(6) The Kirchhoff stress $\tau$ is calculated by

$$
\boldsymbol{\tau}=\sum_{s=1}^{n} f\left(\lambda^{(\mathrm{s})}\right) \mathrm{e}^{-2 \lambda^{(\mathrm{s})}}(\boldsymbol{n} \otimes \boldsymbol{n})+p(\theta) \boldsymbol{I} .
$$

$I$ denotes the identity tensor.

(7) For the slip component, the resolved shear stress is computed based on the Schmid law: $\tau^{(\alpha)}=\boldsymbol{\tau}: \boldsymbol{P}^{(\alpha)}$. For the continuously active slip $\alpha$, the slip deformation rate $\dot{\gamma}^{(\alpha)}$ is calculated by the rate of resolved shear stress $\dot{\tau}^{(\alpha)}$ and the hardening modulus matrix $h_{\alpha \beta}$ based on the eq. (3) [34].

(8) The new symmetric rate of plastic stretching tensor $\left(\tilde{\boldsymbol{D}}^{p}\right)^{n}$ is calculated by $\left(\tilde{\boldsymbol{D}}^{p}\right)^{n}=\sum_{\alpha=1}^{m} \dot{\gamma}^{(\alpha)} \boldsymbol{P}^{(\alpha)}$.

(9) The new symmetric rate of stretching tensor is computed by $(\tilde{\boldsymbol{D}})^{n}=\left(\boldsymbol{D}^{e}\right)^{n}+\left(\tilde{\boldsymbol{D}}^{p}\right)^{n}$, and the computed one $(\tilde{\boldsymbol{D}})^{n}$ is compared with the given one $(\boldsymbol{D})^{n}$. If the condition $\left\|(\tilde{\boldsymbol{D}})^{n}-(\boldsymbol{D})^{n}\right\|<\delta \quad(\delta$ is a small value dependent on the calculation precision) is satisfied, go to the next step; otherwise, assign the value $\left(\tilde{\boldsymbol{D}}^{p}\right)^{n}$ to $\boldsymbol{D}^{p}$ and go back to the second step.

(10) The elasto-plastic damage stiffness $C^{\text {epd }}$ is obtained by eq. (5).

(11) At last, the increase of second Piola-Kirchhoff stress $(\Delta \boldsymbol{T})^{n}$ is calculated by eq. (12), and the total value of second Piola-Kirchhoff stress is obtained by $(\boldsymbol{T})^{n}=(\boldsymbol{T})^{n-1}+(\Delta T)^{n}$.

\section{Simulations}

For the anisotropic 6260-T6 aluminum alloy, the material was tested under uniaxial loading along different material orientations and the material constants were given by Dunand et al. [7]. On the other hand, in the experiments of Luo et al. [28], the flat tensile specimens with circular notches (three different notch radii were used: $R=20 \mathrm{~mm}$, $R=10 \mathrm{~mm}, R=5 \mathrm{~mm}$ ) and specimens with a central hole $(R=5 \mathrm{~mm})$ were employed. All specimens were extracted along three different orientations: the first one was along the extrusion orientation $\left(0^{\circ}\right)$, the second one was along the diagonal orientation $\left(45^{\circ}\right)$, and the last one was along transverse orientation $\left(90^{\circ}\right)$.

The simulations are carried out for the experimental results of Luo et al. [28]. All tensile specimens are discretized using reduced-integration four-node plane stress elements (CPS4R). Considering the symmetry of the specimen geometries and loading conditions, we choose one fourth of the tensile specimens with the thickness of $2 \mathrm{~mm}$ for all 
simulations.

The geometries and mesh grids for the four types of specimens are showed in Figure 1. The left plots are the geometries for each type of specimens, and the right plots are the mesh grids. For the tensile specimens with circular notches, the gage length is $30 \mathrm{~mm}$, and for the specimens with a central hole, the gage length is $20 \mathrm{~mm}$. The width for all tensile specimens is $20 \mathrm{~mm}$.

\subsection{Discrete directions of spring-bundle and slip com- ponents}

The spring-bundle and slip components are discrete in the space. For simplicity in the simulation, 15 independent spring-bundle components are chosen in the plane, as shown in Figure 2(a). Considering that the active slip components can slip along positive or negative directions, we choose 30 independent slip components in the first and second quadrants, as shown in Figure 2(b).
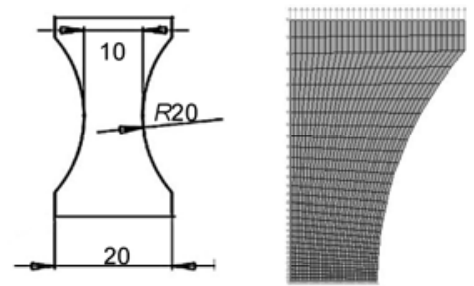

(a)
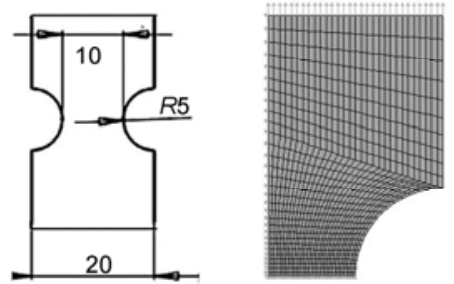

(c)

\subsection{Calibration of model parameters}

In the experiments by Dunand et al. [7], the Young's modulus of $E=63 \mathrm{GPa}$ and the elastic Poisson ratio of $v=0.33$ were given, as well as the yield stress $\sigma_{y s}$ in different material orientations. The average value of the initial uniaxial yield stress is $210 \mathrm{MPa}$.

Before the structural calculation, the pretreatment would be carried out and other model parameters could be determined.

\subsubsection{Pretreatment before the structural calculation}

For each calculated point, the stiffness matrix is determined by the state variables on the components (e.g. deformations history and response). For isotropic material, the initial state variables remain the same in different directions. To simulate the anisotropy of material, we change the state variables before structural calculation.

The pretreatment is completed by using the software
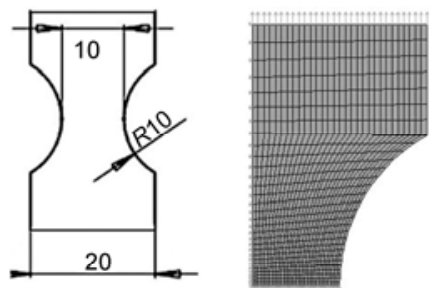

(b)
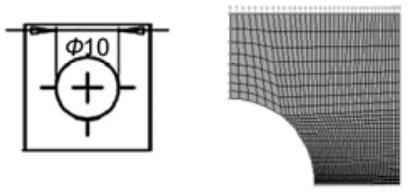

(d)

Figure 1 Geometries and mesh grids for tensile specimens. (a) Specimens with circular notches: $R=20 \mathrm{~mm}$; (b) specimens with circular notches: $R=10 \mathrm{~mm}$; (c) specimens with circular notches: $R=5 \mathrm{~mm}$; (d) specimens with a central hole.
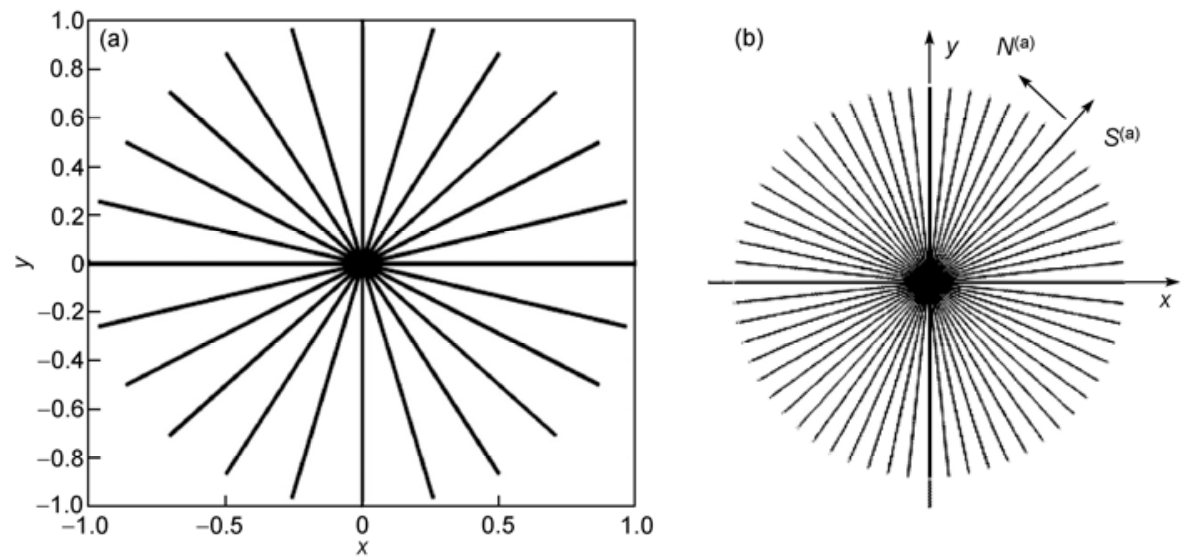

Figure 2 Planar discrete directions of spring-bundle and slip components. (a) Spring-bundle components; (b) slip components. 

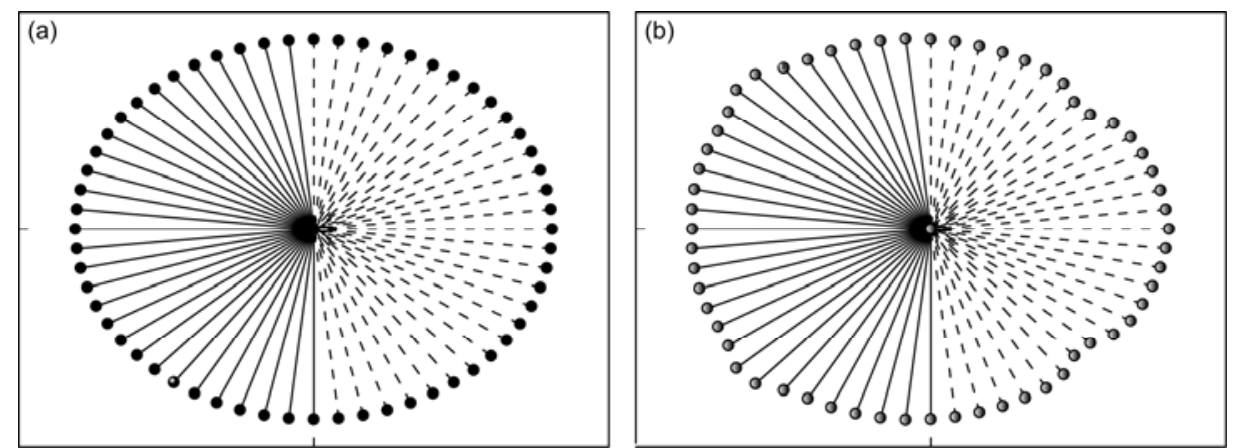

Figure 3 Change of the value of critical resolved shear stress after the pretreatment (solid line: positive direction; dotted line: negative direction). (a) Before pretreatment (isotropic); (b) after pretreatment (anisotropy).

FORTRAN. By setting the initial yield stress $\sigma_{y s}^{0}$ as 210 $\mathrm{MPa}$, each calculated point would experience a tensile loading to $211 \mathrm{MPa}$ (the yield stress in the extrusion orientation) and a linear unloading to zero-stress state.

The state variables on slip components would change according to their own directions, such as the critical resolved shear stress and slip deformation. For example, the change of critical resolved shear stress in different directions after the pretreatment is shown in Figure 3. The length of line represents the critical value. The solid line and dotted line represent the critical value of positive and negative directions respectively.

The anisotropy is reflected by the variety of state variables on the discrete components. Then the value of the current state variables would be saved as the initial value for the structural calculation.

\subsubsection{Calibration of slip components}

(1) The value of initial critical resolved shear stress $\tau_{ \pm c r}^{0}$ is determined by the average value of initial uniaxial yield stress as $\tau_{ \pm c r}^{0}=210 \mathrm{MPa} / 2=105 \mathrm{MPa}$ [34]. After the pretreatment, the critical resolved shear stress would differ from one another.

(2) The hardening rule in the present model includes isotropic and kinematic hardening [34], and it is defined as:

$$
h_{\alpha \beta}=h_{i}^{(\alpha, \beta)}+h_{k}^{(\alpha, \beta)}
$$

where $h_{i}^{(\alpha, \beta)}$ represents isotropic hardening, and $h_{k}^{(\alpha, \beta)}$ represents kinematic hardening.

From the comparison between the maximum tension stress (about $240 \mathrm{MPa}$ ) and the initial yield stress (about $210 \mathrm{MPa}$ ) in the experiments [7], the Aluminum in the experiments is considered as a kind of very low work hardening material. Based on the investigation of Liu et al. [34] and the experimental results of Khan et al. [39-41], the kinematic hardening dominates the hardening rule for low work hardening materials. So the isotropic hardening is not considered in the present work.

The hardening modulus matrix $h_{\alpha \beta}$ is expressed as:

$$
\begin{aligned}
h_{\alpha \beta} & =h_{k}^{(\alpha, \beta)} \\
& =h_{k l}^{(\alpha, \beta)} \boldsymbol{P}^{(\alpha)}: \boldsymbol{P}^{(\beta)}+\left(h_{k s}^{(\alpha)}-h_{k l}^{(\alpha, \beta)}\right) \delta_{\alpha \beta} \boldsymbol{P}^{(\alpha)}: \boldsymbol{P}^{(\beta)},
\end{aligned}
$$

where $h_{k s}^{(\alpha)}$ and $h_{k l}^{(\alpha, \beta)}$ denote self and latent hardening respectively.

Considering that the exponential curve is similar to the macroscopic stress-strain curve, we express the self hardening parameter $h_{k s}^{(\alpha)}$ as:

$$
h_{k s}^{(\alpha)}=2 c_{k} m_{k}\left(\left|\gamma^{(\alpha)}\right|+\gamma_{0}\right)^{m_{k}-1},
$$

where $c_{k}$ and $m_{k}$ are the kinematic hardening parameters of the model, and $\gamma_{0}$ is determined by $\gamma_{0}=\left(\frac{\tau_{+c r}^{0}}{c_{k}}\right)^{\frac{1}{m_{k}}}$.

Considering that the earlier active slip components affect the later ones and based on the symmetry of the hardening matrix, we express the latent hardening parameter $h_{k l}^{(\alpha, \beta)}$ as:

$$
h_{k l}^{(\alpha, \beta)}=q \min \left(h_{k s}^{(\alpha)}, h_{k s}^{(\beta)}\right),
$$

where $q$ is the latent hardening parameter.

The response of the slip component can be expressed as:

$$
\tau^{(\alpha)}=c_{k}\left(|\gamma|+\gamma_{0}\right)^{m_{k}} .
$$

The response curve of the continuously active slip component is revealed in Figure 4.

(3) Considering that the Bauschinger effect parameter $b$ and the latent hardening parameter $q$ affect the change of critical resolved shear stress in the pretreatment, we determine these two parameters by the difference of yield stress between the extrusion orientation $\left(0^{\circ}\right)$ and other orientations $\left(45^{\circ}, 90^{\circ}\right)$.

(4) The kinematic hardening parameters $c_{k}$ and $m_{k}$ are determined by fitting the stress-strain curve under uniaxial loading. Figure 5 shows the comparison of stress-strain 


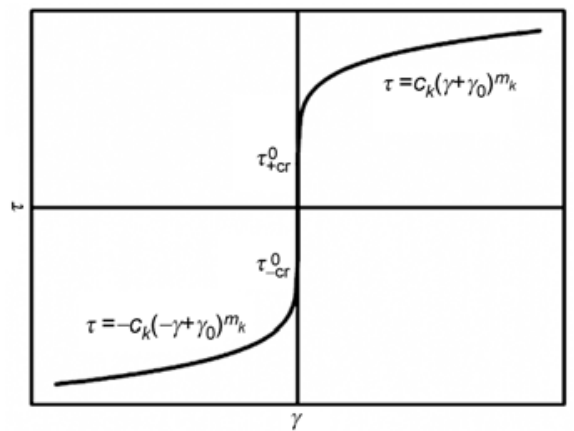

Figure 4 Response curve of the continuously active slip component.

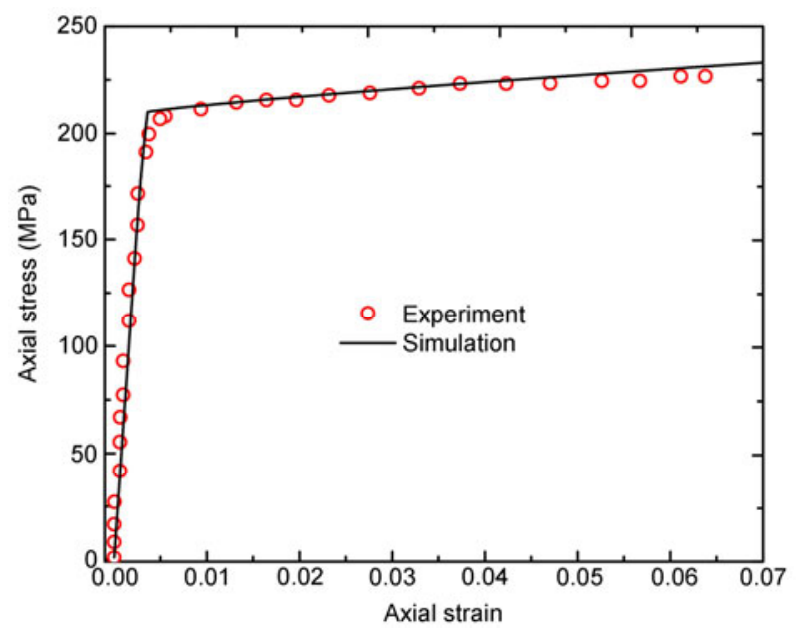

Figure 5 Comparison of stress-strain curve in uniaxial loading between the simulation and experimental results.

curve between the simulation and experimental results.

\subsubsection{Calibration of spring-bundle components}

Considering that the stiffness of spring-bundle component decreases exponentially, we express the response of the spring-bundle component as:

$$
f\left(\lambda^{(s)}\right)= \begin{cases}\left(H_{0} \lambda_{0}+H_{0} k\left(1-\mathrm{e}^{\frac{-\lambda_{+h}^{(s)}+\lambda_{0}}{k}}\right)\right) \frac{\lambda^{(s)}}{\lambda_{+h}^{(s)}}, & \lambda_{+h}^{(s)}>\lambda_{0}, \\ H_{0} \lambda, & -\lambda_{0} \leqslant \lambda_{ \pm h}^{(s)} \leqslant \lambda_{0}, \\ \left(-H_{0} \lambda_{0}-H_{0} k\left(1-\mathrm{e}^{\frac{\lambda_{-h}^{(s)}+\lambda_{0}}{k}}\right)\right) \frac{\lambda^{(s)}}{\lambda_{-h}^{(s)}}, & \lambda_{-h}^{(s)}<-\lambda_{0},\end{cases}
$$

where $H_{0}$ is the initial stiffness of spring-bundle component, which is determined by Young's module and Poisson's ratio. $\lambda_{0}$ is the damage threshold value in tension loading. $\lambda_{ \pm h}^{(s)}$ is the deformation history. $k$ is the parameter that determines the rate of damage development of the spring-bundle component. Figure 6 is the response curve of the springbundle component.

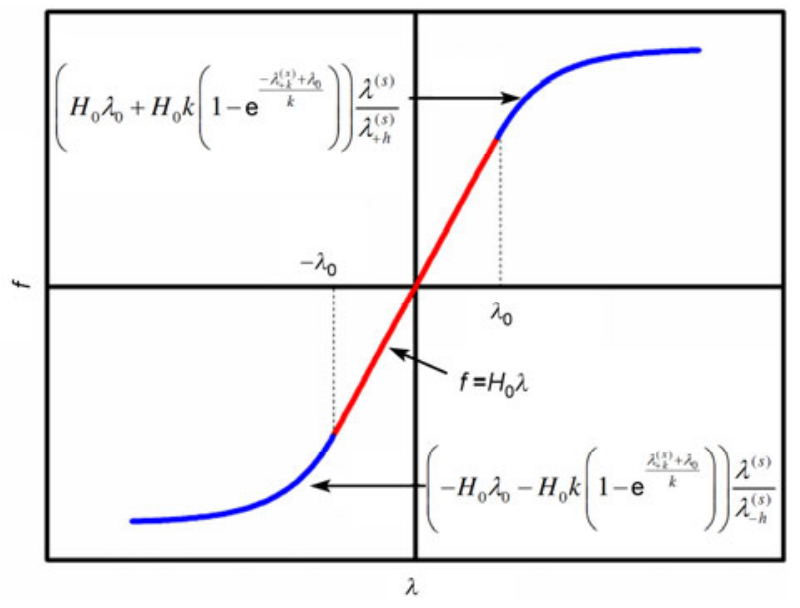

Figure 6 Response curve of the spring-bundle component.

Comparing the tangent stiffness tensor in eq. (2) with the generalized Hooke's law, we obtain the initial stiffness $H_{0}$ of spring-bundle component by the initial Young's modulus and Poisson ratio [34]. In the plane stress condition, $H_{0}$ is calculated by $H_{0}=\frac{4 E}{\pi(1+v)}$. The damage threshold value $\lambda_{0}$ and the parameter $k$ are determined by fitting the force-displacement curves along the extrusion orientation $\left(0^{\circ}\right)$ in the experiments by Luo et al. [28].

\subsubsection{Calibration of cubage component}

The damage of cubage component is not considered in the present work, so the force response $p(\theta)$ can be calculated by the initial Young's modulus and Poisson ratio [34]:

$$
p(\theta)=\frac{E(3 v-1)}{2(1+v)(1-v)} \theta .
$$

All parameters for simulation are listed in Table 1 and kept the same irrespective of the specimen geometries.

\subsection{Simulation results}

The comparisons for tensile specimens with circular notches between simulation and experimental results are shown in Figure 7 and these for tensile specimens with a central hole are revealed in Figure 8. 'FD' represents force-displacement, and the local Log strain at the center of the planes is taken into account.

Figure 9 is the comparisons of the axial strain for the tensile specimens in extrusion orientation between the simulation and experimental results. The left plots are the experimental results measured by Digital Image Correlation from the specimen surface, and the contour plots on the right are simulations of logarithmic axial strain. It can be

Table 1 Parameters for simulations

\begin{tabular}{ccccccccc}
\hline$E(\mathrm{GPa})$ & $v$ & $\lambda_{0}$ & $k$ & $\tau_{ \pm c r}^{0}(\mathrm{MPa})$ & $c_{k}(\mathrm{MPa})$ & $m_{k}$ & $q$ & $b$ \\
\hline 63 & 0.33 & 0.0024 & 0.017 & 105 & 5000 & 0.1 & 0.1 & 1.0 \\
\hline
\end{tabular}



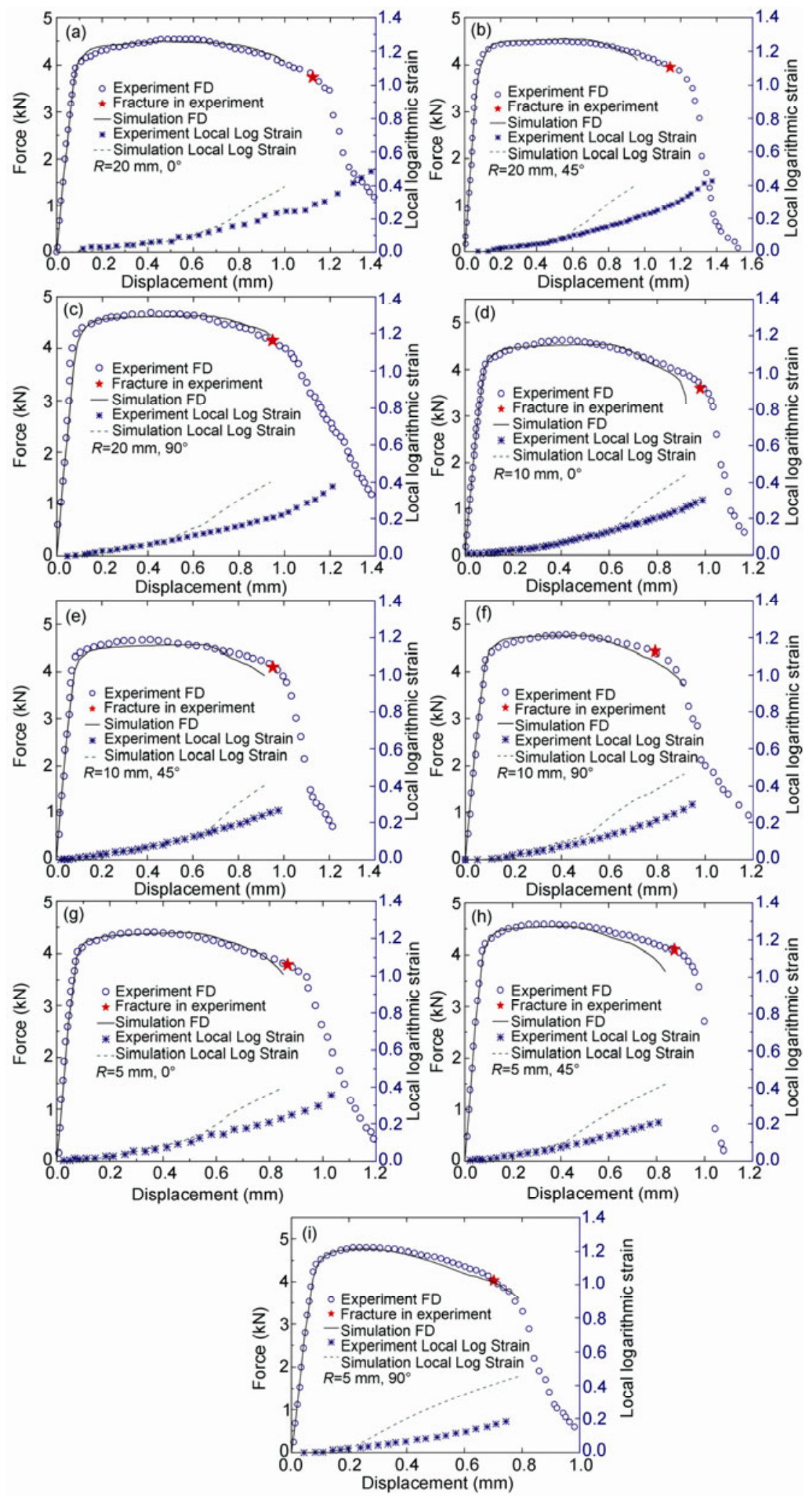

Figure 7 (Color online) Comparisons of experimental and simulation results in force-displacement response and central logarithmic axial strain evolution for the tensile specimens with circular notches. (a) $R=20 \mathrm{~mm}, \alpha=0^{\circ}$; (b) $R=20 \mathrm{~mm}, \alpha=45^{\circ}$; (c) $R=20 \mathrm{~mm}, \alpha=90^{\circ}$; (d) $R=10 \mathrm{~mm}, \alpha=0^{\circ}$; (e) $R=10 \mathrm{~mm}, \alpha=45^{\circ}$; (f) $R=10 \mathrm{~mm}, \alpha=90^{\circ}$; (g) $R=5 \mathrm{~mm}, \alpha=0^{\circ}$; (h) $R=5 \mathrm{~mm}, \alpha=45^{\circ}$; (i) $R=5 \mathrm{~mm}, \alpha=90^{\circ}$. 

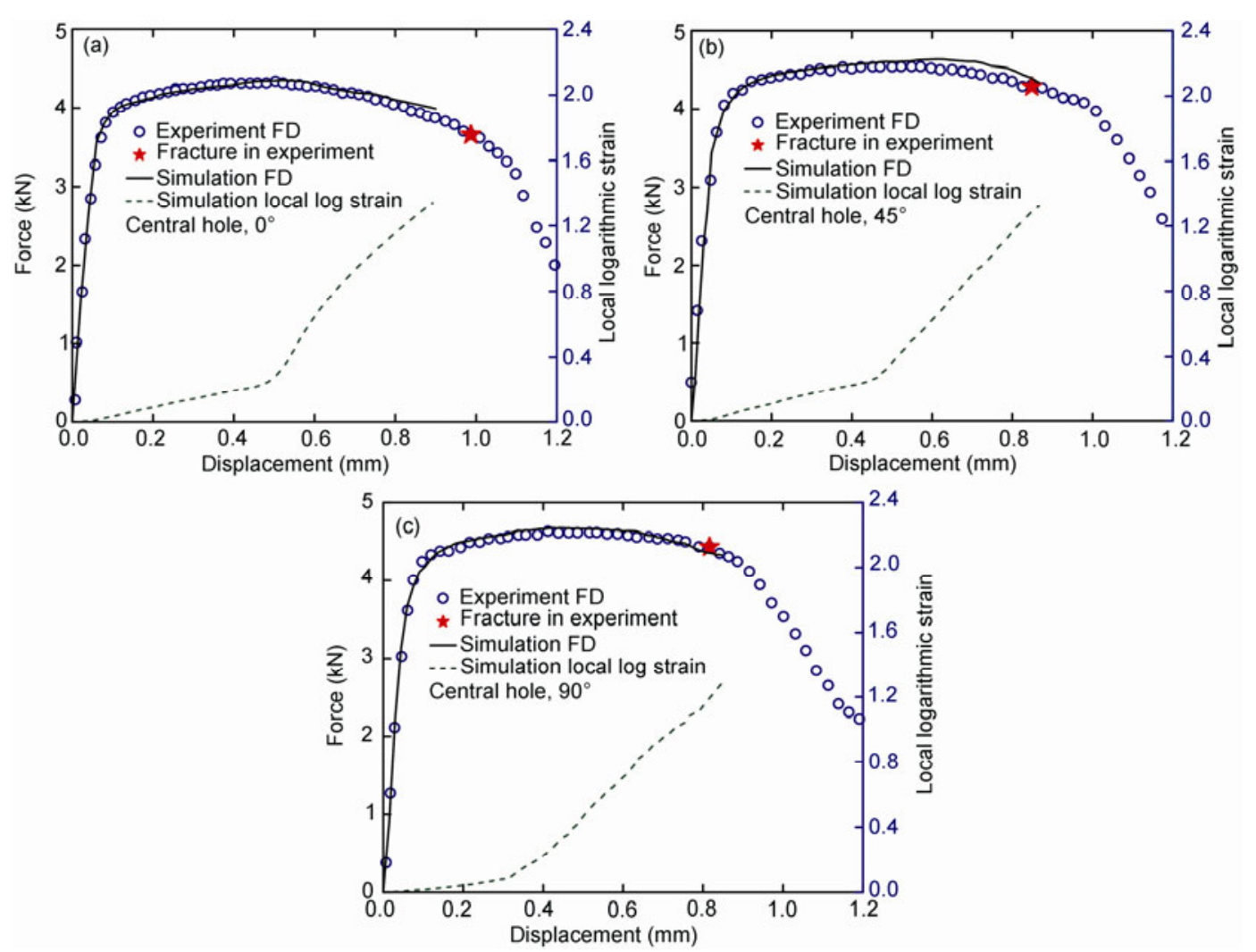

Figure 8 (Color online) Comparisons of the force-displacement response between experimental and simulation results and the evolution of central logarithmic axial strain for the tensile specimens with a central hole. (a) $\alpha=0^{\circ}$; (b) $\alpha=45^{\circ}$; (c) $\alpha=90^{\circ}$.

found that the strain localization is near the center of the specimens both in simulation and experimental results.

From the comparisons in Figures 7-9, it can be obtained that the model is able to describe the elasto-plastic-damage behavior of materials.

\section{Discussion}

The force-displacement response curves for the tensile specimens are showed in the Figure 10. Same with the experiment results, a noticeable anisotropy is observed.

In the present work, the plasticity is reflected by the slip components. The loading and unloading processes in the pretreatment make the state variables on slip components changing according to their own directions. Moreover, the change of critical resolved shear stress is affected by Bauschinger effect parameter $b$ and the latent hardening parameter $q$, which makes the yield stress diverse in different material orientations. Then in the structural calculation, the plastic deformation induced anisotropy is reflected by the slip components.

On the other hand, the damage is determined by deformations on the spring-bundle components, which depend on the directions of themselves. Then in the structural calculation, the damage evolution in different spring-bundle com- ponents reflects the anisotropic damage.

For the specimens in different material orientations, the macroscopic anisotropy is reflected by the state variables on the components naturally.

\section{Conclusions}

The present work focuses on the elasto-plastic-damage behavior of anisotropic aluminum alloys under finite deformation by using a physical mechanism based constitutive model. The main conclusions of this paper are summarized below.

(1) The constitutive equation is transformed into a linear format and applied in the structural calculation using the user subroutine (VUMAT) of ABAQUS. For the same material, the parameters are the same in all cases and have their physical meanings.

(2) For tensile specimens from the extruded 6260-T6 aluminum alloy profile, the comparisons between the simulation and experimental results show the capacity of the present model to describe the elasto-plastic-damage behavior of anisotropic materials.

(3) The anisotropy is explained based on the present constitutive theory. Since the spring-bundle and slip components discrete all over the space, and the state variables on 


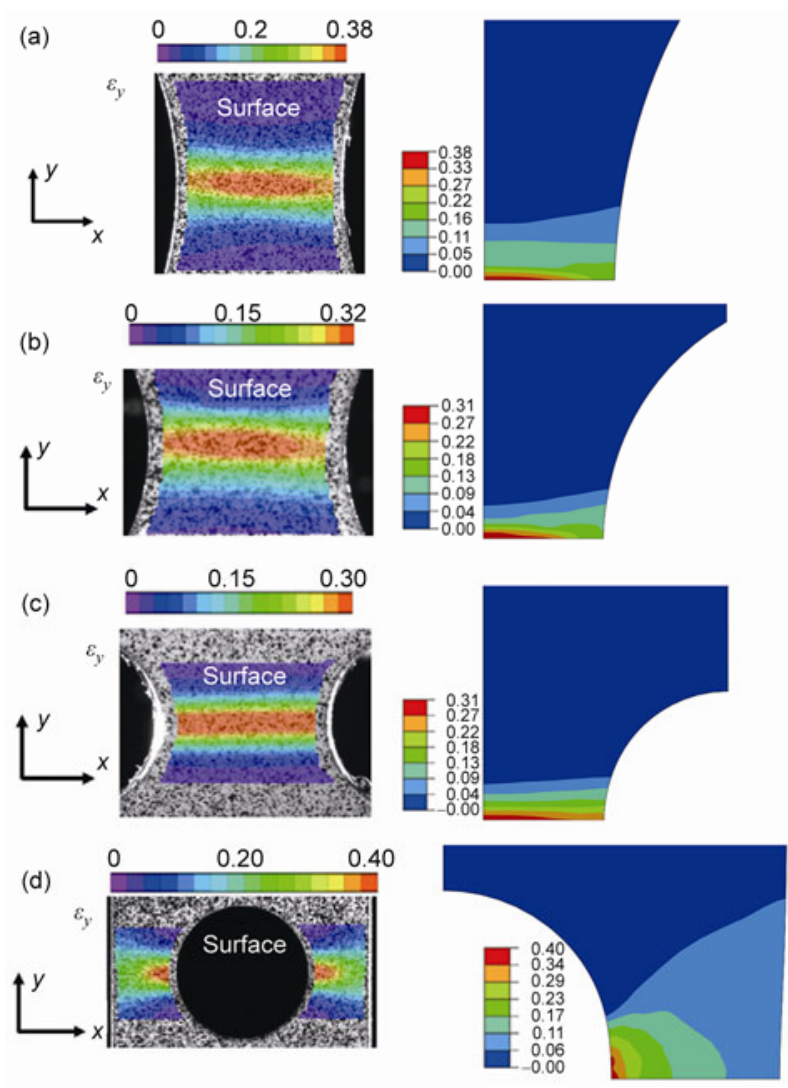

Figure 9 Comparisons of simulation and experimental results in the axial strain for tensile specimens in extrusion orientation. (a) Specimen with circular notches: $R=20 \mathrm{~mm}$; (b) specimen with circular notches: $R=10 \mathrm{~mm}$; (c) specimen with circular notches: $R=5 \mathrm{~mm}$; (d) specimen with a central hole.
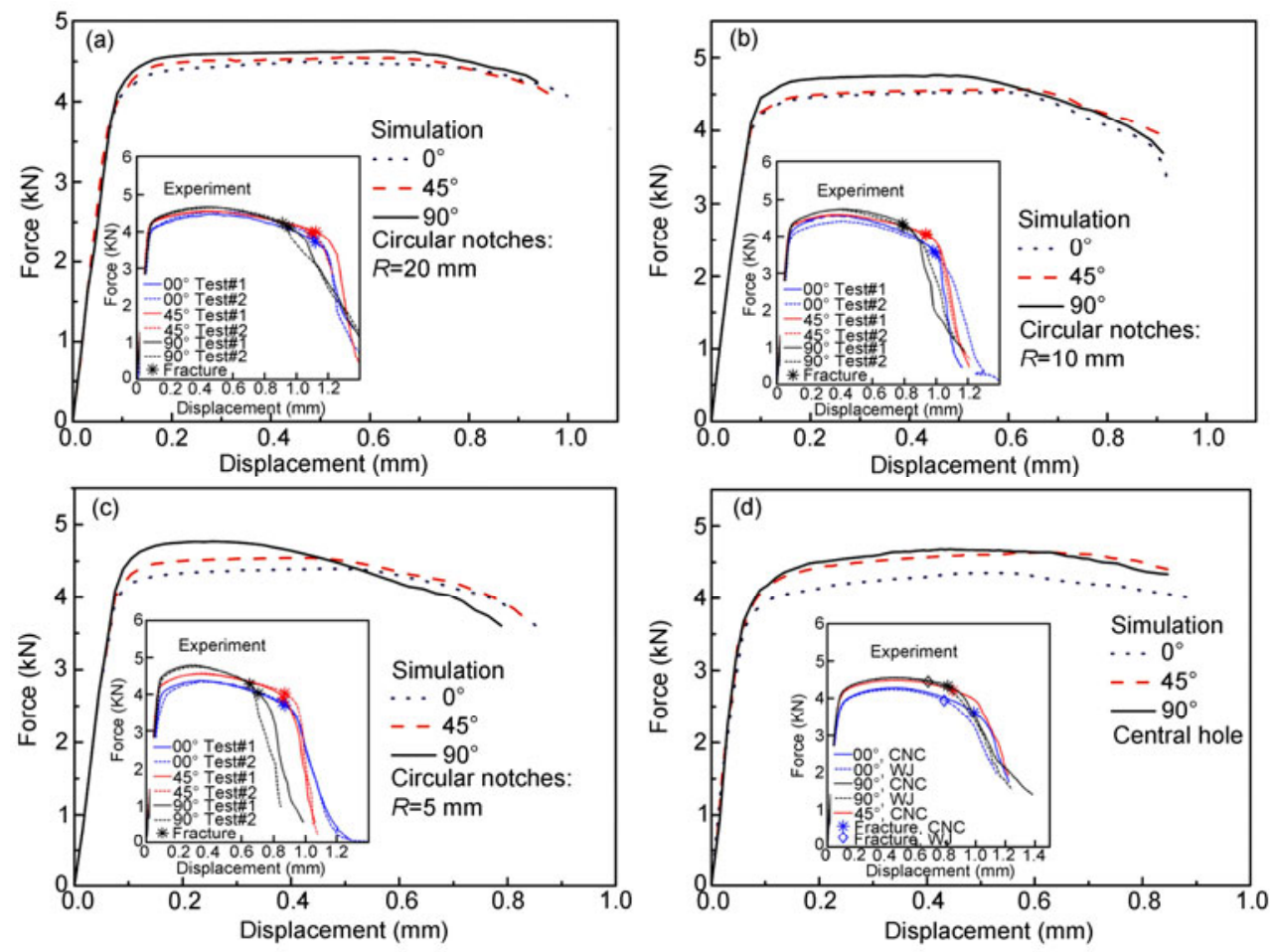

Figure 10 (Color online) Simulation results in the force-displacement response for tensile specimens. (a) Specimens with circular notches: $R=20$ mm; (b) specimens with circular notches: $R=10 \mathrm{~mm}$; (c) specimens with circular notches: $R=5 \mathrm{~mm}$; (d) specimens with a central hole. 
these two kinds of components change according to their directions, the macroscopic property is naturally reflected by the evolution of state variables on the components.

The authors thank Professor HONG YouShi and ZHAO YaPu for helpful suggestions. This work was supported by the National Natural Science Foundation of China (Grant No. 11021262) and the Fundamental Research Funds for the Central Universities (Grant No. WUT: 2013-IV-021).

1 Hill R. A theory of the yielding and plastic flow of anisotropic metals. Proc R Soc Lond, 1948, 193: 281-297

2 Hill R. The Mathematical Theory of Plasticity. Oxford: Clarendon Press, 1950

3 Barlat F, Becker R C, Hayashida Y, et al. Yielding description for solution strengthened aluminum alloys. Int J Plast, 1997, 13(4): 385-401

4 Barlat F, Brem J C, Yoon J W, et al. Plane stress yield function for aluminum alloy sheets-part 1: theory. Int J Plast, 2003, 19(9): 1297-1319

5 Barlat F, Lege D J, Brem J C. A six-component yield function for anisotropic materials. Int J Plast, 1991, 7(7): 693-712

6 Barlat F, Maeda Y, Chung K, et al.Yield function development for aluminum alloy sheets. J Mech Phy Solids, 1997, 45(11-12): $1727-1763$

7 Dunand M, Maertens A P, Luo M, et al. Experiments and modeling of anisotropic aluminum extrusions under multi-axial loading-Part I: Plasticity. Int J Plast, 2012, 36(0): 34-49

8 Garmestani H, Kalidindi S R, Williams L, et al. Modeling the evolution of anisotropy in Al-Li alloys: Application to Al-Li 2090-T8E41. Int J Plast, 2002, 18(10): 1373-1393

9 Zamiri A, Pourboghrat F, Barlat F. An effective computational algorithm for rate-independent crystal plasticity based on a single crystal yield surface with an application to tube hydroforming. Int $\mathbf{J}$ Plast, 2007, 23(7): 1126-1147

10 Lemaitre J. A continuous damage mechanics model for ductile fracture. J Eng Mater Technol, 1985, 107(1): 83-89

11 Krajcinovic D, Fonseka G U. The continuous damage theory of brittle materials, Part 1: General Theory. J Appl Mech, 1981, 48(4): 809-815

12 Krajcinovic D, Mastilovic S. Some fundamental issues of damage mechanics. Mech Mater, 1995, 21(3): 217-230

13 Litewka A, Debinski J. Load-induced oriented damage and anisotropy of rock-like materials. Int J Plast, 2003, 19(12): 2171-2191

14 Simo J C, Ju J W. Strain- and stress-based continuum damage models-I. Formulation. Int J Solid Struct, 1987, 23(7): 821-840

15 Simo J C, Ju J W. Strain- and stress-based continuum damage models-II. Computational aspects. Int J Solid Struct, 1987, 23(7): 841-869

$16 \mathrm{Ju}$ J, Lee X. Micromechanical damage models for brittle solids. Part I: Tensile Loadings. J Eng Mech, 1991, 117(7): 1495-1514

17 Krajcinovic D, Fanella D. A micromechanical damage model for concrete. Eng Frac Mech, 1986, 25(5-6): 585-596

18 Lee X, Ju J. Micromechanical damage models for brittle solids. Part II: Compressive loadings. J Eng Mech, 1991, 117(7): 1515-1536

19 Zhu Q, Kondo D, Shao J, et al. Micromechanical modelling of anisotropic damage in brittle rocks and application. Int J Rock Mech Min Sci, 2008, 45(4): 467-477

20 Peeters B, Hoferlin E, Van Houtte P, et al. Assessment of crystal plasticity based calculation of the lattice spin of polycrystalline metals for FE implementation. Int J Plast, 2001, 17(6): 819-836
21 Kowalczyk K, Gambin W. Model of plastic anisotropy evolution with texture-dependent yield surface. Int J Plast, 2004, 20(1): 19-54

22 Gao H, Klein P. Numerical simulation of crack growth in an isotropic solid with randomized internal cohesive bonds. J Mech Phy Solids, 1998, 46(2): 187-218

23 Miller R, Ortiz M, Phillips R, et al. Quasicontinuum models of fracture and plasticity. Eng Frac Mech, 1998, 61(3-4): 427-444

24 Huang Z, Dui G, Yang S. A new interpretation of internal-variable theory in finite thermo-viscoelasticity. Sci China-Phys Mech Astron, 2013, 56(3): 610-616

25 Huang Z P. Fundamentals of Continuum Mechanics. Beijing: Higher Education Press, 2003

26 Wang Z Q, Dui G S. Two-point constitutive equations and integration algorithms for isotropic-hardening rate-independent elastoplastic materials in large deformation. Int J Numer Meth Eng, 2008, 75(12): $1435-1456$

27 Wang Z, Zhao Y. Thermo-hyperelastic models for nanostructured materials. Sci China-Phys Mech Astron, 2011, 54(5): 948-956

28 Luo M, Dunand M, Mohr D. Experiments and modeling of anisotropic aluminum extrusions under multi-axial loading - Part II: Ductile fracture. Int J Plast, 2012, (32-33): 36-58

29 Deng S C, Liang N G, Lu H X. The elasto-damage theory of the components assembling model. Sci China Ser G-Phys Mech Astron, 2009, 52(01): 1-12

30 Deng S C, Liu J X, Liang N G, et al. Validation of component assembly model and extension to plasticity. Theor Appl Fract Mech, 2007, 47(3): 244-259

31 Deng S C, Liu J X, Zhang J, et al. Component assembling model and its application to quasi-brittle damage. Theor Appl Fract Mech, 2006, 46(3): 232-242

32 Zhang J, Liang N G, Deng S C, et al. Study of the damage-induced anisotropy of quasi-brittle materials using the component assembling model. Int J Damage Mech, 2008, 17(3): 197-221

33 Liu F, Fu Q, Chen C, et al. An elasto-plastic damage constitutive theory based on pair functional potentials and slip mechanism. Chin J Aeronaut, 2010, 23(6): 686-697

34 Liu F, Fu Q, Chen C, et al. An elasto-plastic damage constitutive theory and its prediction of evolution of subsequent yield surfaces and elastic constants. Int J Plast, 2011, 27(9): 1355-1383

35 Fu Q, Liu F, Chen C, et al. A study on the evolution of subsequent yield surfaces with finite deformation (In Chinese). Eng Mech, 2011, 28(11): 23-30

36 Fu Q, Liu F, Zhang J, et al. A physically motivated model for the evolution of subsequent yield surfaces (In Chinese). Chin J Theor Appl Mech, 2010, 42(5): 880-888

37 Chen $\mathrm{C}$, Liu F, Fu Q, et al. Investigation of subsequent yield surface evolution under biaxial tension (in Chinese). Acta Aeronaut Astronaut Sin, 2012, 33(12): 2230-2239

38 Ericksen J L. The cauchy and born hypotheses for crystals. In: Phase Transformations and Material Instabilities in Solids. New York: Academic Press, 1984

39 Khan A S, Kazmi R, Pandey A, et al. Evolution of subsequent yield surfaces and elastic constants with finite plastic deformation. Part-I: A very low work hardening aluminum alloy (A16061-J6511). Int J Plast, 2009, 25(9): 1611-1625

40 Khan A S, Pandey A, Stoughton T. Evolution of subsequent yield surfaces and elastic constants with finite plastic deformation. Part III: Yield surface in tension-tension stress space (Al 6061-T 6511 and annealed 1100 Al). Int J Plast, 2010, 26(10): 1432-1441

41 Khan A S, Pandey A, Stoughton T. Evolution of subsequent yield surfaces and elastic constants with finite plastic deformation. Part II: A very high work hardening aluminum alloy (annealed $1100 \mathrm{Al}$ ). Int J Plast, 2010, 26(10): 1421-1431 